(D) Gislaine Barbosa Bezerra ${ }^{1}$

(D) Cristiane Ferreira Rodrigues ${ }^{2}$

(DRenata Brum Martucci ${ }^{1,3}$

1 Instituto Nacional do Câncer José de Alencar Gomes da Silva. Departamento de Nutrição. Rio de Janeiro, RJ, Brasil.

2 Instituto Nacional do Câncer José de Alencar Gomes da Silva. Departamento de Fonoaudiologia.

Rio de Janeiro, RJ, Brasil.

3 Universidade do Estado do Rio de Janeiro, Instituto de Nutrição, Departamento de Nutrição

Aplicada. Rio de Janeiro, RJ, Brasil.

Correspondência

Gislaine Barbosa Bezerra

gislaine.proativa@yahoo.com.br

\section{Caracterização do perfil alimentar e nutricional dos sobreviventes de câncer de cavidade oral e grau de adesão às recomendações de prevenção ao câncer emitidas pelo Fundo Mundial de Pesquisa de câncer e Instituto Americano de Pesquisa de Câncer}

Characterization of the food intake and nutritional profile of oral cavity cancer survivors and the adherence degree to cancer prevention recommendations issued by the World Cancer Research Fund and the American Institute for Cancer Research Institute

\begin{abstract}
Resumo
Introdução: Sobreviventes do câncer de cavidade oral (SCCO) em controle normalmente apresentam inúmeras sequelas derivadas do tratamento oncológico, o que pode comprometer sua alimentação e, por extensão, o atendimento às diretrizes de alimentação e peso adequado emitidas pelo Fundo Mundial para Pesquisa do Câncer/Instituto Americano de Pesquisa para o Câncer (WCRF/AICR). Objetivo: Avaliar o perfil alimentar e o estado nutricional de sobreviventes de câncer de cavidade oral e verificar o grau de sua adesão, as diretrizes de alimentação saudável e peso corporal emitidas pela WCRF / AICR. Método: Estudo transversal que avaliou o estado nutricional pela Avaliação Subjetiva Global produzida pelo paciente (ASG-PPP) e índice de massa corporal (IMC) de 20 pacientes. Dados como condição social e histórico clínico foram coletados em prontuário. O consumo alimentar foi avaliado por meio de um diário alimentar de 30 dias. Os dados alimentares e o peso corporal receberam um escore de pontos conforme maior ou menor grau de adesão às recomendações WCRF / AICR. Resultados: $80 \%$ dos pacientes encontravam-se com desnutrição moderada ou suspeita de desnutrição pela ASG-PPP. Pelo IMC, 10\% dos participantes estavam desnutridos, 45\% eutróficos e 35\% apresentavam sobrepeso. Foi encontrado escore médio 2,64 \pm 0.55 de adesão às recomendações de peso adequado e alimentação saudável, demonstrando atendimento de menos de $50 \%$ das recomendações WCRF / AICR. Conclusão: Os pacientes SCCO do presente estudo apresentam risco de desnutrição ou estão com desnutrição moderada; e possuem alta sintomatologia, que impacta negativamente na ingestão alimentar e na adesão às recomendações da WCRF / AICR.
\end{abstract}

Palavras-chave: Câncer de cavidade oral. Xerostomia. Sobreviventes de câncer. Alimentação saudável.

Abstract

Introduction: Survivors of oral cavity cancer (OCC) under control usually have numerous sequelae resulting from oncological treatment, which can compromise their diet and, by extension, meet the guidelines for diet and proper weight issued by the World Cancer Research Fund International-WCRF and the American Institute for Cancer 
Research-AICR. Objective: To evaluate the dietary profile and nutritional status of oral cancer survivors and verify the degree of their adherence, the guidelines for healthy eating and body weight issued by WCRF/AICR. Method: Cross-sectional study which assessed the nutritional status by the Subjective Global Assessment produced by the patient (PG-SGA) and body mass index (BMI) of 20 patients. Data such as social condition and clinical history were collected from medical records. Food consumption was assessed using a 30-day food diary. Food data and body weight received a score of points according to a greater or lesser degree of adherence to WCRF/AICR recommendations. Results: $80 \%$ of patients were moderately malnourished or suspected of malnutrition by PG-SGA. According to BMI 10\% of participants were malnourished, 45\% eutrophic and 35\% were overweight. An average score of $2.64 \pm$ 0.55 adherence to the recommendations of adequate weight and healthy eating was found, showing compliance with less than 50\% of the WCRF/AICR recommendations. Conclusion: Survivor patients of OCC patients from the present study are at risk of malnutrition or have moderate malnutrition; and have high symptomology, which negatively impacts food intake and adherence to recommendations of WCRF/AICR.

Keywords: Oral cavity cancer. Xerostomia. Cancer survivors. Healthy eating. 


\section{INTRODUÇÃO}

Sobreviventes de câncer, por definição, são todas as pessoas que recebem diagnóstico de câncer, independentemente do estágio da doença ou se iniciaram, finalizaram ou continuam em tratamento oncológico. ${ }^{1}$

O número de sobreviventes de câncer vem aumentando proporcionalmente à epidemia desta doença, em decorrência de progressos na área de diagnóstico e tratamento. Dados da terceira edição do Atlas de Câncer indicam que, em 2018, existiam aproximadamente 43,8 milhões de pessoas vivendo com o diagnóstico de câncer nos últimos cinco anos. Para esse mesmo ano, as projeções indicavam 18 milhões de novos casos de câncer. 0 aumento de sobreviventes de câncer representa um desafio mundial para os sistemas de saúde e previdenciário, considerando as limitações de ordem física, social e mental muitas vezes causadas pelo tratamento oncológico nesta população. No Brasil, estima-se que mais de 162 mil sobreviventes de câncer de todas as idades e sexo estejam vivendo com alguma deficiência causada pela doença e tratamento. ${ }^{2}$

O câncer de cavidade oral (CCO) é o quarto tipo mais incidente em homens e o décimo segundo mais frequente entre todos os tipos de câncer. Sua incidência tem aumentado nos últimos anos, principalmente entre os jovens, devido ao aumento da infecção por vírus HPV. Segundo o INCA (Instituto Nacional de Câncer), foram estimados 15.190 novos casos em 2020-2022. ${ }^{3}$

O tratamento para esta doença depende do tipo histológico, estadiamento da doença e performance status do paciente; envolve recursos cirúrgicos, quimioterápicos e radioterápicos, que podem ser aplicados unicamente ou de forma multimoda. ${ }^{4}$ É um grande desafio garantir uma nutrição adequada para pacientes sobreviventes de câncer de cavidade oral (SCCO), já que as taxas de sequelas para este tipo de câncer são elevadas, variando entre 40 e 90\% e afetando órgãos que fazem parte do sistema digestório. ${ }^{5}$

Promover uma alimentação adequada aos sobreviventes de câncer é uma recomendação do Fundo Mundial de Pesquisa de Câncer (WCRF) / Instituto Americano de Pesquisa de Câncer (AICR). Tal recomendação é respaldada por estudos que demonstram que essa população apresenta risco maior de desenvolver um segundo tumor, o qual pode ser reduzido se fatores de proteção como manutenção da atividade física, alimentação e peso adequado forem adotados. Neste sentido, o WCRF publicou, em 1997, a primeira edição das dez diretrizes para dieta, nutrição, atividade física na prevenção do câncer aplicável à população geral e também aos sobreviventes de câncer. Em 2018, WCRF / AICR lançaram a terceira revisão dessas recomendações, mantendo as dez diretrizes que continuam aplicáveis aos sobreviventes de câncer. ${ }^{6}$

Diversos estudos demonstram que a adesão às recomendações de alimentação, peso saudável e atividade física possui potencial de prevenção de câncer para cada um ponto de escore.-12 Contudo, a despeito desses benefícios, poucos são os estudos sobre adesão a essas recomendações na população em geral e, sobretudo, em pacientes sobreviventes de câncer, principalmente em SCCO.

Os pacientes SCCO normalmente apresentam inúmeras sequelas derivadas do tratamento oncológico que podem limitar sua ingestão alimentar e, assim, comprometer seu estado nutricional. ${ }^{12-14}$ Dentre as diversas sequelas do tratamento oncológico que ocorrem nos pacientes SCCO, destaca-se a xerostomia, prevalente nesta população e impactante na sua qualidade de vida. 5,15,16 Neste sentido, a proposta deste estudo foi avaliar o perfil alimentar e o estado nutricional dos sobreviventes em controle de câncer de cavidade oral e verificar o grau de adesão dessa população às diretrizes de alimentação saudável e peso corporal emitidas por WCRF / AICR. 


\section{MÉTODO}

\section{Tipo de estudo}

Estudo transversal realizado com SCCO atendidos no ambulatório de Fonoaudiologia do Hospital de Câncer I do Instituto Nacional do Câncer José Alencar Gomes da Silva. O estudo foi aprovado pelo Comitê de Ética sob nº 90318418.9.0000.5274, em 10/09/2018. A amostra foi realizada por conveniência com 20 pacientes derivados do estudo "Efeitos do uso de flavorizante na qualidade de vida e nos achados funcionais em pacientes com queixa de xerostomia submetidos a tratamento radioterápico para tumores de cavidade oral".

Os critérios de inclusão foram: ser maior de 18 anos, ter concluído o tratamento oncológico há pelo menos 180 dias, estar em acompanhamento no ambulatório de fonoaudiologia e possuir diagnóstico de xerostomia. Foram excluídos do estudo pacientes que apresentassem parotidite, tivessem realizado cirurgia de remoção de glândulas salivares, síndrome Sjögren e / ou que faziam uso prévio continuado de medicamentos contendo atropina, drogas anti-histamínicas, anfetaminas e fenotiazínicos. Os pacientes que aceitaram participar do estudo assinaram o termo de consentimento livre e esclarecido (TCLE).

\section{Dados sociais e clínicos}

No prontuário médico dos pacientes, foram coletados dados clínicos relativos ao diagnóstico e estadiamento da doença, tratamento oncológico empregado, tempo livre de doença e histórico de comorbidades, além de presença de casos de câncer na família. Foram verificados também, no prontuário, dados relativos ao consumo de álcool e fumo, peso habitual antes da doença, bem como dados sociais (escolaridade e profissão). Tais dados foram registrados em uma ficha para coleta de dados sociais e clínicos.

\section{Avaliação do Perfil Nutricional e Alimentar}

A avaliação nutricional foi conduzida por métodos objetivos e subjetivos, no ato da admissão dos pacientes na pesquisa. A avaliação nutricional foi realizada por meio dos seguintes instrumentos: Avaliação Subjetiva Global produzida pelo próprio paciente (ASG-PPP) e índice de massa corporal (IMC).

Foi utilizada a ASG-PPP proposta por Ottery, ${ }_{1}^{17}$ traduzida e validada em português por Gonzalez et al. ${ }^{18} \mathrm{e}$ extraído da plataforma http://pt-global.org/?page_id=13<<, acesso em 03 de abril 2019.17,18 Para tanto, foram assinalados na caixa 1 do formulário ASG-PPP dados referidos pelo paciente acerca da alteração do seu peso corporal no último mês e nos seis meses anteriores. Os pacientes foram questionados acerca da presença de sintomas de impacto na ingestão alimentar nas últimas duas semanas, os quais foram devidamente assinalados na caixa 3. Os pacientes também foram indagados sobre ocorrência de modificações dietéticas no último mês (caixa 2) além da sua condição na realização de atividades e função (caixa 4). A partir do preenchimento da primeira parte da ASG-PPP, foi obtido um escore numérico que forneceu recomendações para aplicação da terapia nutricional. Após determinação dos escores, foi preenchida a segunda parte da ASG-PPP pela coleta de dados referentes à história clínica do paciente, além do grau de estresse metabólico, e assinalados os dados informados pelos pacientes na caixa 4. Realizou-se, com auxílio da ASG-PPP, uma avaliação física no paciente, a fim de classificar as reservas musculares, adiposas e hídricas dos grandes grupamentos musculares em três categorias: 0 - sem déficit; 1 - déficit leve; 2 - déficits moderado; e 3 - déficit grave.

Após a coleta de todos esses dados, realizou-se avaliação geral da condição nutricional do paciente, classificando-o como: A - bem nutrido; B - moderadamente desnutrido ou em risco de desnutrição; ou C gravemente desnutrido, de acordo com a gravidade das repercussões nutricionais verificadas pelos dados relativos 
ao percentual de alteração de peso, modificação permanente da ingestão alimentar, diminuição da capacidade física, além do grau de perda de tecidos.

\section{Medidas antropométricas}

\section{Registro do peso}

Os pacientes, portando roupas leves, foram posicionados em pé, descalços, no centro da balança digital marca Filizola ${ }^{\circledR}$, com capacidade de $150 \mathrm{~kg}$ e precisão de 100 g, devidamente zerada. Após estabilização da balança, o pesquisador registrou em formulário próprio o peso do paciente.

\section{Registro da altura do paciente}

A medição de altura foi realizada por meio de estadiômetro móvel acoplado à balança de marca Filizola ${ }^{\circledR}$, com altura $2 \mathrm{~m}$ e precisão de $1 \mathrm{~mm}$. Para tanto, posicionou-se o paciente em pé, descalço com os calcanhares juntos, costas retas e os braços estendidos ao lado do corpo, olhar fixo para horizonte, encostado na balança de costas para o visor. A haste móvel do estadiômetro foi posicionada sobre a cabeça do paciente pelo pesquisador. Após aferição da altura, o dado foi registrado em formulário próprio.

\section{Determinação do IMC}

A partir dos dados registrados de altura e peso dos pacientes, foi determinado o IMC a partir da fórmula: IMC $\left(\mathrm{Kg} / \mathrm{m}^{2}\right)$ : peso kg/ altura $\mathrm{m}^{2}$. A classificação do IMC foi realizada segundo a faixa etária do indivíduo adulto, conforme a classificação de referência de IMC proposto pela Organização Mundial da Saúde (OMS). ${ }^{19}$

\section{Avaliação do perfil alimentar}

Em formulário próprio, o pesquisador realizou um recordatório alimentar de 24 horas de cada paciente; e ainda, cada paciente recebeu um manual fotográfico de quantificação alimentar conforme Crispim et al. ${ }^{20}$ e um diário alimentar para ser preenchido durante 30 dias.

Foi estipulada uma meta individual de necessidade de ingestão de calorias e proteínas por meio da fórmula de bolso descrita no Consenso Brasileiro de Nutrição Oncológica. ${ }^{21}$ Em seguida, determinou-se o quantitativo em termos de calorias e proteínas consumido pelos pacientes por meio dos dados alimentares descritos no recordatório alimentar com o apoio do software brasileiro NUMAX ${ }^{\circledR}$ NUMAX Desenvolvimento de sistema Ltda ME. Brasil, versão 1.2.56. Para avaliação da adequação de consumo, foi estabelecida a seguinte relação: \% adequação às metas calóricas e proteicas = consumo individual de calorias e ou proteínas X100 / pela meta de consumo individual de calorias e / ou proteínas.

Em relação aos dados de adesão às recomendações de nutrição e consumo alimentar emitidas por WCRF / AICR, foi empregada a metodologia proposta por Shams-White et al. ${ }^{22}$ Resumidamente, foram extrapolados dos diários alimentares de cada paciente os códigos de cada alimento, listados conforme descrito no Manual Fotográfico de Quantificação Alimentar. A partir desse código, verificou-se o per capita de alimentos consumidos dos seguintes grupos alimentares: alimentos ultraprocessados, bebidas açucaradas, carnes vermelhas, carnes processadas, verduras, frutas e legumes, consumo de álcool e fibras. Os per capitas diários do consumo desses alimentos foram incluídos em uma planilha eletrônica Excel ${ }^{\circledR}$, onde foi aplicado um escore de pontos, conforme grupo de alimento e per capita consumido, segundo o quadro 1. 
Quadro 1. Resumo dos escores de pontos atribuídos ao atendimento de cada item de recomendação de operacionalização das diretrizes de alimentação e peso saudável emitido por WCRF / AIRC, 2018.

\begin{tabular}{|c|c|c|}
\hline \multirow[t]{2}{*}{ Recomendação WCRF / AICR } & OPERACIONALIZAÇÃO & PONTOS \\
\hline & $\mathrm{IMC}\left(\mathrm{kg} / \mathrm{m}^{2}\right)$ & Pontos \\
\hline \multirow[t]{3}{*}{ 1-Mantenha peso saudável } & $18.5-24.9$ & 1 \\
\hline & $25-29.9$ & 0,5 \\
\hline & $<18.5$ ou $\geq 30$ & 0 \\
\hline \multirow[t]{8}{*}{$\begin{array}{l}\text { 2- Faça uma dieta rica em cereais } \\
\text { integrais, legumes, frutas e feijões }\end{array}$} & $\begin{array}{l}\text { Frutas e vegetais } \\
\text { Consumo (g/dia) }\end{array}$ & Pontos \\
\hline & 400 & 0.5 \\
\hline & $200-<400$ & 0.25 \\
\hline & $<200$ & 0 \\
\hline & Total de fibras (g) dia & Pontos \\
\hline & 30 & 0.5 \\
\hline & $15-<30$ & 0.25 \\
\hline & $<15$ & 0 \\
\hline \multirow{4}{*}{$\begin{array}{l}\text { 3- Limite o consumo de fast food e outros } \\
\text { alimentos processados ricos em gordura, } \\
\text { amido e açúcares }\end{array}$} & $\begin{array}{l}\text { Porcentagem do total de kcal de alimentos } \\
\text { ultraprocessados (aUPFs): }\end{array}$ & Pontos \\
\hline & Tercil 1 & 1.0 \\
\hline & Tercil 2 & 0.5 \\
\hline & Tercil 3 & 0 \\
\hline \multirow{4}{*}{$\begin{array}{l}\text { 4- Limite o consumo de carne vermelha e } \\
\text { carne processada }\end{array}$} & $\begin{array}{l}\text { Total carne vermelha (g/semana) e carne } \\
\text { processada (g/semana): }\end{array}$ & Pontos \\
\hline & $\begin{array}{r}\text { Carne vermelha }<500 \mathrm{~g} \text { e processada } 21 \mathrm{~g} \\
\text { (semana) }\end{array}$ & 1.0 \\
\hline & $\begin{aligned} \text { Carne vermelha }< & 500 \mathrm{~g} \text { e processada 21-100g } \\
& \text { (semana) }\end{aligned}$ & 0.5 \\
\hline & $\begin{aligned} \text { Carne vermelha } & >500 \mathrm{~g} \text { e carne processada } \\
& \geq 100 \mathrm{~g}\end{aligned}$ & 0 \\
\hline \multirow{4}{*}{ 5- Limite o consumo de álcool } & Total em g etanol dia & Pontos \\
\hline & 0 (nenhum consumo) & 1.0 \\
\hline & $>0-\leq 28 \mathrm{~g}$ homens e $\leq 14 \mathrm{~g}$ mulheres & 0.5 \\
\hline & $>28 \mathrm{~g}$ homens e $>14 \mathrm{~g}$ mulheres & 0 \\
\hline \multirow{4}{*}{$\begin{array}{l}\text { 6- Limite o consumo de bebidas } \\
\text { açucaradas }\end{array}$} & Total bebidas adoçadas (g/dia): & Pontos \\
\hline & 0 (nenhum consumo) & 1.0 \\
\hline & $>0-\leq 250$ & 0.5 \\
\hline & $>250$ & 0 \\
\hline
\end{tabular}

Fonte: Adaptado de Shams-White et al., 2019.

Com relação à recomendação de peso saudável, o IMC de cada paciente foi categorizado com base nas faixas de classificação de IMC definido pela OMS, e também incluído em planilha eletrônica Excel ${ }^{\circledR}$. A partir da classificação do IMC, foi dado um escore de ponto conforme o quadro 1.

Após determinação de cada escore de consumo alimentar e peso saudável de cada participante da pesquisa, foi realizado um somatório de todos os pontos obtidos dos escores, recomendações e nutrição e peso definidos por WCR / AICR. A adesão plena às recomendações perfaria um escore máximo de seis pontos. Não há um ponto de corte para adesão às recomendações, sendo recomendado o maior escore possível.

\section{Dados estatísticos}

As variáveis categóricas foram descritas por meio de frequência absoluta e relativa (percentual). As variáveis contínuas foram descritas por meio de média e desvio padrão. As associações entre variáveis categóricas foram descritas por meio dos testes Exato de Fisher e Qui-Quadrado de Pearson. 
A aderência das variáveis contínuas à distribuição normal foi testada pelo teste de Shapiro-Wilk. Quando confirmadas, as diferenças nas medidas de tendência central foram testadas por meio do teste $t$ para amostras independentes. Quando não confirmadas, foram testadas pelo teste de Mann-Whitney. O nível de significância adotado foi de 5\%, e o software utilizado foi o R Core Team 2020.

\section{Resultados}

Na tabela 1, encontra-se a caraterização social e clínica dos 20 pacientes que participaram da pesquisa. A média de tempo livre de doença foi de 619 dias.

Tabela 1. Caracterização social e clínica dos pacientes acompanhados pelo ambulatório de fonoaudiologia que iniciaram o estudo. Rio de Janeiro-RJ, 2020.

\begin{tabular}{|c|c|c|}
\hline Variáveis & $\mathrm{N}$ & $\%$ \\
\hline \multicolumn{3}{|l|}{ Sexo } \\
\hline Masculino & 12 & 60 \\
\hline feminino & 8 & 40 \\
\hline \multicolumn{3}{|l|}{ Faixa etária } \\
\hline Adulto & 12 & 60 \\
\hline Maior de 60 anos & 8 & 40 \\
\hline \multicolumn{3}{|l|}{ Tipo de câncer } \\
\hline CEC orofaringe & 5 & 25 \\
\hline CEC laringe & 1 & 5 \\
\hline CEC Palato duro & 2 & 10 \\
\hline CEL língua & 8 & 40 \\
\hline CEC Amígdala & 4 & 20 \\
\hline \multicolumn{3}{|l|}{ Estádio da doença } \\
\hline । & 2 & 10 \\
\hline$\|$ & 3 & 15 \\
\hline III & 8 & 40 \\
\hline IV & 7 & 35 \\
\hline \multicolumn{3}{|c|}{ Tipo de tratamento empregado } \\
\hline Quimioterapia & 11 & 55 \\
\hline Radioterapia & 20 & 100 \\
\hline Cirurgia & 12 & 60 \\
\hline \multicolumn{3}{|l|}{ Grau de instrução } \\
\hline Sem instrução & 1 & 5 \\
\hline Ensino fundamental & 9 & 45 \\
\hline Ensino médio & 9 & 45 \\
\hline Ensino superior & 1 & 5 \\
\hline \multicolumn{3}{|l|}{ Raça } \\
\hline Negra / pardo & 17 & 85 \\
\hline Branca & 3 & 15 \\
\hline Amarelos & 0 & 0 \\
\hline \multicolumn{3}{|l|}{ Casos de câncer familiar } \\
\hline Sim & 7 & 35 \\
\hline Não & 13 & 65 \\
\hline
\end{tabular}

Legenda: $\mathrm{n}$ - frequência absoluta. \% - frequência relativa percentual. 
Pacientes adultos e do sexo masculino foram os mais prevalentes, representando $60 \%$ da amostra. Em relação ao estágio da doença, 75\% dos pacientes apresentaram diagnóstico de doença avançada em estágios III e IV. O CEC de língua foi o tipo mais incidente de câncer, afetando 40\% da amostra. Com relação à escolaridade, houve a mesma proporção (45\%) de pacientes que apresentavam primeiro grau ou segundo grau completo.

Em relação às comorbidades preexistentes, 20\% dos pacientes apresentam diabetes e 40\%, hipertensão arterial. Além destas doenças, 40\% dos pacientes apresentaram outras comorbidades, como hipotireoidismo, doenças cardíacas e gastrites (dados não apresentados).

Com relação a hábitos de vida, nesta amostra o tabagismo foi o hábito mais prevalente nos pacientes, representando 61,5\%, seguido de ingestão de bebidas alcóolicas, presente em 53,8\% da amostra.

Na tabela 2, é possível avaliar a classificação do estado nutricional avaliado pelo IMC antes da doença e após concluído tratamento oncológico. Pelo IMC habitual, observa-se que antes da doença havia uma prevalência maior de sobrepeso e obesidade, afetando 65\% da amostra estudada. Não havia pacientes antes da doença com diagnóstico de desnutrição. Após o tratamento oncológico, observa-se que 10\% dos pacientes apresentavam quadro de desnutrição, 45\% dos pacientes foram classificados como eutróficos e 35\% com sobrepeso e obesidade, pelos critérios do IMC atual.

Tabela 2. Caracterização do perfil alimentar e nutricional dos pacientes que iniciaram o estudo acompanhados pelo ambulatório de fonoaudiologia. Rio de Janeiro-RJ, 2020.

\begin{tabular}{|c|c|c|}
\hline Variáveis & $\mathrm{N}$ & $\%$ \\
\hline \multicolumn{3}{|l|}{$N^{0}$ de refeições realizadas } \\
\hline 3 & 5 & 25 \\
\hline 4 & 9 & 45 \\
\hline 5 & 6 & 30 \\
\hline \multicolumn{3}{|l|}{ Consistência da dieta } \\
\hline Branda & 9 & 45 \\
\hline Pastosa & 11 & 55 \\
\hline Semilíquida & 0 & 0 \\
\hline Liquida & 0 & 0 \\
\hline \multicolumn{3}{|l|}{ Atendimento das metas recomendações de de ingestão calórica } \\
\hline Abaixo & 5 & 25 \\
\hline Pleno atendimento & 0 & 0 \\
\hline Excesso & 15 & 75 \\
\hline \multicolumn{3}{|l|}{ Atendimento das metas de ingestão proteica } \\
\hline Abaixo & 9 & 45 \\
\hline Atende & 0 & 0 \\
\hline Excesso & 11 & 55 \\
\hline \multicolumn{3}{|l|}{ Classificação do IMC habitual antes da doença } \\
\hline Baixo-peso & 0 & 0 \\
\hline Eutrofia & 7 & 35 \\
\hline Sobrepeso/Obesidade & 13 & 65 \\
\hline \multicolumn{3}{|l|}{ Classificação do IMC atual após a doença } \\
\hline Baixo-peso & 2 & 10 \\
\hline Eutrofia & 9 & 45 \\
\hline Sobrepeso/Obesidade & 9 & 45 \\
\hline \multicolumn{3}{|l|}{ Classificação atual da ASG PPP } \\
\hline Bem nutrido - A & 4 & 20 \\
\hline Moderadamente desnutrido/risco de desnutrição - B & 16 & 80 \\
\hline Desnutrição grave - C & 0 & 0 \\
\hline
\end{tabular}


Tabela 2. Caracterização do perfil alimentar e nutricional dos pacientes que iniciaram o estudo acompanhados pelo ambulatório de fonoaudiologia. Rio de Janeiro-RJ, 2020. (Cont)

\begin{tabular}{|c|c|c|}
\hline & $\mathrm{N}$ & $\%$ \\
\hline \multicolumn{3}{|c|}{ Pontuação total da ASG PPP } \\
\hline Até 3 pontos & 0 & 0 \\
\hline 4 a 8 pontos & 4 & 20 \\
\hline 9 pontos & 16 & 80 \\
\hline \multicolumn{3}{|c|}{ Mudança de peso nas duas últimas semanas conforme ASG PPP } \\
\hline Diminuiu & 3 & 15 \\
\hline Ficou igual & 7 & 35 \\
\hline Aumentou & 10 & 50 \\
\hline
\end{tabular}

Problemas que impedem de se alimentar nas últimas duas semanas (ASG-PPP)

Sem problemas para me alimentar

Sem apetite, apenas sem vontade de comer

5

Náuseas

Obstipação (intestino preso)

Feridas na boca

Coisas tem gosto estranho ou não tem gosto

Problemas para engolir

Vômitos

Diarreia

Boca seca

Cheiros incomodam

Me sinto rapidamente satisfeito

Cansaço e fadiga

Dor

Outros

$\begin{array}{cc}1 & 5 \\ 10 & 50 \\ 4 & 20 \\ 5 & 25 \\ 1 & 5 \\ 15 & 75 \\ 12 & 60 \\ 1 & 5 \\ 1 & 5 \\ 20 & 100 \\ 3 & 15 \\ 8 & 40 \\ 5 & 25 \\ 6 & 30 \\ 8 & 40\end{array}$

Ingestão alimentar no último mês comparada com minha alimentação habitual

A mesma coisa

Mais que o habitual

Menos que o habitual

$\begin{array}{cc}1 & 5 \\ 0 & 0 \\ 19 & 95\end{array}$

\section{Atualmente minha ingestão alimentar é}

A mesma comida (sólida) em menor quantidade que o habitual $\quad 12 \quad 60$

A mesma comida (sólida) em pouca quantidade $\quad 5 \quad 25$

Apenas alimentos líquidos $\quad 3 \quad 15$

Apenas suplementos nutricionais $\quad 0 \quad 0$

Muito pouca quantidade de qualquer alimento $\quad 0 \quad 0$

Apenas alimentação por sonda ou pela veia $\quad 0 \quad 0$

Atividades e função: no último mês, de um modo geral eu consideraria a minha atividade (função) como

Normal, sem nenhuma limitação

Não totalmente normal, mas capaz de manter quase todas as atividades normais

210

Sem disposição para a maioria das coisas, mas ficando na cama ou na

cadeira menos da metade do dia

$2 \quad 10$

Capaz de fazer pouca atividade e passando a maior parte do dia na cadeira ou na cama

Praticamente acamado, raramente fora da cama

$10 \quad 50$

Exame físico - perda muscular

\begin{tabular}{lcc}
0 & 10 & 50 \\
1 & 6 & 30 \\
2 & 4 & 20 \\
3 & 0 & 0 \\
\hline Exame físico - reserva de gordura & & 40 \\
0 & 8 & 40 \\
1 & 8 & 20 \\
3 & 4 & 0 \\
\hline
\end{tabular}


Tabela 2. Caracterização do perfil alimentar e nutricional dos pacientes que iniciaram o estudo acompanhados pelo ambulatório de fonoaudiologia. Rio de Janeiro-RJ, 2020. (Cont)

\begin{tabular}{llc}
\hline \multicolumn{1}{c}{ Variáveis } & $\mathrm{N}$ & $\%$ \\
\hline Classificação da perda de peso em um mês & & 0 \\
$\geq 10 \%$ & 0 & 0 \\
$5-9,9 \%$ & 0 & 0 \\
$3-4,9 \%$ & 0 & 10 \\
$2-2,9 \%$ & 2 & 0 \\
$0-1,9 \%$ & 0 & \\
\hline
\end{tabular}

Legenda: $\mathrm{n}$ - frequência absoluta. \% - frequência relativa percentual.

Em relação à classificação do estado nutricional avaliado pela ASG-PPP, 80\% dos participantes encontravamse em risco nutricional. A maioria apresentou sintomas com mais de nove pontos na pontuação total, o que indica uma necessidade urgente de conduta para a melhora dos sintomas e/ou opções de intervenção nutricional, conforme descrito nessa ferramenta. Dos sintomas listados na ASG-PPP, a xerostomia (boca seca) foi o mais prevalente em toda a população estudada. Além deste sintoma, outros referidos pelos pacientes foram: disgeusia (alteração do paladar, com pacientes referindo que alimentos têm gosto estranho ou não têm gosto), atingindo 75\% dos pacientes; hiporexia (falta de vontade para se alimentar) em 50\% da amostra; e disfagia (problemas para engolir) em $60 \%$ da população.

Em relação à ingestão alimentar, 90\% dos pacientes referiram menor ingestão no último mês, comparada com a alimentação habitual, além de sintomas de cansaço, não conseguindo realizar suas atividades habituais. Referiram, no entanto, aumento de peso.

Avaliando os dados extraídos do diário alimentar e do recordatório alimentar, observa-se ingestão mais prevalente de alimentos na consistência pastosa (55\%), com excesso de calorias e proteínas (75\% e 55\%, respectivamente).

Dos pacientes que entregaram o diário alimentar ( $n=13)$, conforme pode ser verificado na tabela 3, 46,2\% não alcançaram metade do escore de pontos de atendimento das recomendações de alimentação e peso corporal emitidas por WCRF / AICR. A média de escore desta população foi de 2,64 \pm 0.55 , não atendendo, portanto, nem metade das recomendações, que têm como pontuação máxima 6 pontos (IMC médio de 24,96 $\pm 5.41 \mathrm{~kg} / \mathrm{m}^{2}$ ). Proporção igual de pacientes atingiu escore de 3,0 a 3,75 pontos, que equivale a atendimento de $50 \%$ a $62,5 \%$ das recomendações.

Tabela 3. Distribuição do percentual de atendimento aos escores de alimentação saudável e peso corporal emitidos por WCRF / AICR dos pacientes acompanhados pelo ambulatório de fonoaudiologia. Rio de Janeiro-RJ, 2020.

\begin{tabular}{ccc}
\hline $\begin{array}{c}\text { Categorização do escore de atendimento peso corporal e } \\
\text { alimentação WCRF / IARC }\end{array}$ & $\mathrm{N}$ & $\%$ \\
\hline$<2$ pontos & 5 & 38,5 \\
$2.1-2,9$ pontos & 1 & 7,7 \\
$3,0-3,75$ & 6 & 46,1 \\
$3.8-4,5$ & 1 & 7,7 \\
\hline
\end{tabular}

Legenda: $\mathrm{n}$ - frequência absoluta. \% - frequência relativa percentual

Verificando o escore dos grupos alimentares estratificados conforme as recomendações WCRF / AICR, conforme a tabela 4, pode-se verificar que 93\% dos participantes apresentam alto consumo de carne processada 
ou carne vermelha, não atendendo, portanto, à recomendação de consumo limitado de carne vermelha e processada. Em relação às calorias da dieta provenientes de alimentos ultraprocessados ou com alta densidade calórica, observa-se que mais de dois terços das calorias advindos do consumo de alimentos ultraprocessados fazem parte da alimentação de 53,8\% dos pacientes estudados. Importante salientar que, pelos critérios das recomendações de WCRF / AICR, as bebidas açucaradas são classificadas à parte dos alimentos ultraprocessados, a fim de evitar dupla penalidade no escore. Em relação ao consumo de bebidas açucaradas, observa-se que 61,5\% dos pacientes apresentam consumo de até $250 \mathrm{ml}$ ao dia, e 38,5\% deles consomem mais de $250 \mathrm{ml}$ ao dia.

Tabela 4. Distribuição de pacientes que completaram o estudo acompanhados pelo ambulatório de fonoaudiologia, em relação ao atendimento ao escores de recomendações de WCRF / AICR para prevenção do câncer. Rio de Janeiro-RJ, 2020.

\begin{tabular}{|c|c|c|c|c|}
\hline Recomendações WCRF/AICR & Operacionalização & Escore & $\mathrm{N}$ & $\%$ \\
\hline \multirow{4}{*}{ 1-Mantenha o peso saudável } & $I M C\left(\mathrm{~kg} / \mathrm{m}^{2}\right)$ & Escore & $\mathrm{N}$ & $\%$ \\
\hline & $18.5-24.9$ & 1 & 7 & 53,8 \\
\hline & $25-29.9$ & 0,5 & 2 & 15,4 \\
\hline & $<18.5$ ou $\geq 30$ & 0 & 4 & 30,8 \\
\hline \multirow{8}{*}{$\begin{array}{l}\text { 2- Faça uma dieta rica em } \\
\text { cereais integrais, legumes, frutas } \\
\text { e feijões }\end{array}$} & Consumo (g/dia) frutas e vegetais & Escore & N & $\%$ \\
\hline & 400 & 0.5 & 01 & 7,7 \\
\hline & $200-<400$ & 0.25 & 04 & 30,8 \\
\hline & $<200$ & 0 & 08 & 61,5 \\
\hline & Total de fibras (g) dia & Pontos & N & $\%$ \\
\hline & 30 & 0.5 & 01 & 7,7 \\
\hline & $15-<30$ & 0.25 & 04 & 30,8 \\
\hline & $<15$ & 0 & 08 & 61,5 \\
\hline \multirow{5}{*}{$\begin{array}{l}\text { 3- Limite o consumo de fast food } \\
\text { e outros alimentos processados } \\
\text { ricos em gordura, amido e } \\
\text { açúcares }\end{array}$} & $\begin{array}{l}\text { Porcentagem do total de kcal de alimentos } \\
\text { ultraprocessados (aUPFs): }\end{array}$ & Pontos & N & $\%$ \\
\hline & Tercil 1 & 1.0 & 06 & 46.2 \\
\hline & Tercil 2 & 0.5 & 06 & 46.2 \\
\hline & Tercil 3 & 0 & 01 & 7.6 \\
\hline & $\begin{array}{c}\text { Consumo carne vermelha (g/semana) e carne } \\
\text { processada (g/semana): }\end{array}$ & Pontos & N & $\%$ \\
\hline \multirow{2}{*}{$\begin{array}{l}\text { 4- Limite o consumo de carne } \\
\text { vermelha e carne processada }\end{array}$} & Carne vermelha < 500g e processada $21 \mathrm{~g}$ (semana) & 1.0 & 01 & 7,7 \\
\hline & $\begin{aligned} \text { Carne vermelha }< & 500 \mathrm{~g} \text { e processada } 21-100 \mathrm{~g} \\
& (\text { semana) }\end{aligned}$ & 0.5 & 03 & 23,1 \\
\hline \multirow{6}{*}{ 5- Limite o consumo de álcool } & Carne vermelha $>500 \mathrm{~g}$ e carne processada $\geq 100 \mathrm{~g}$ & 0 & 09 & 69,2 \\
\hline & Consumo total em g etanol /dia & Pontos & N & $\%$ \\
\hline & 0 (nenhum consumo) & 1.0 & 13 & $100 \%$ \\
\hline & $>0-\leq 28 \mathrm{~g}$ homens e $\leq 14 \mathrm{~g}$ mulheres & 0.5 & 0 & 0 \\
\hline & >28g homens e >14g mulheres & 0 & 0 & 0 \\
\hline & Consumo bebidas adoçadas (g/dia): & Pontos & $\mathrm{N}$ & $\%$ \\
\hline \multirow{3}{*}{$\begin{array}{l}\text { 6- Limite o consumo de bebidas } \\
\text { açucaradas }\end{array}$} & 0 (nenhum consumo) & 1.0 & 0 & 0 \\
\hline & $>0-\leq 250 \mathrm{~g}$ & 0.5 & 8 & 61,5 \\
\hline & $>250 \mathrm{~g}$ & 0 & 5 & 38,5 \\
\hline
\end{tabular}

Em relação ao consumo de frutas, verduras, legumes e fibras, somente $7,7 \%$ dos pacientes atendem à recomendação de consumo per capita médio de $400 \mathrm{~g}$ ao dia e $30 \mathrm{~g}$ de fibra. Os pacientes deste estudo não apresentaram consumo de álcool no período de acompanhamento, sendo este o único critério plenamente atendido por $100 \%$ dos participantes. Nenhum paciente atendeu plenamente às recomendações de alimentação saudável e peso corporal saudável, não obtendo assim o escore de seis pontos. Não houve diferença estatística 
entre as variáveis estudadas, comparadas com o escore de adesão abaixo ou acima de 50\% das recomendações WCRF / AICR (tabela 5).

Tabela 5. Avaliação do grau de atendimento das recomendações WCRF / AICR em relação às variáveis clínicas e nutricionais dos pacientes acompanhados pelo ambulatório de fonoaudiologia que completaram o estudo. Rio de Janeiro-RJ, 2020.

\begin{tabular}{lccc}
\hline & \multicolumn{2}{c}{ CLASSIF ESCORE WCRF } & p-valor \\
\cline { 2 - 3 } & \multicolumn{1}{c}{50} & $51-90$ & $0,408^{\top}$ \\
Faixa etária & $50(16)$ & $57,8(6,2)$ & $0,414^{\mathrm{W}}$ \\
Tempo (dias) livre da doença & $277,5(230,3)$ & $535,6(577,9)$ & $0,199^{\mathrm{W}}$ \\
No refeições & $4,5(1)$ & $3,8(0,7)$ & $0,410^{\top}$ \\
Escore Total ASG & $15,3(5)$ & $12,8(4,7)$ & $1,000^{\mathrm{W}}$ \\
IMC atual & $26,5(9,4)$ & $24,3(2,9)$ & $0,954^{\top}$ \\
IMC habitual antes da doença & $29(10,4)$ & $29,4(3,9)$ &
\end{tabular}

\section{DISCUSSÃO}

Neste estudo, a prevalência de CCO foi maior no sexo masculino, bem como as incidências de outras comorbidades, consumo de álcool e fumo. Estes achados estão em concordância com outros estudos que tratam do tema. ${ }^{23-25}$ Diabetes e hipertensão foram as morbidades mais prevalentes na população estudada. Em relação ao diabetes, estudos demonstram associação deste com a incidência de câncer de cabeça e pescoço. ${ }^{26-28}$

Observam-se repercussões nutricionais advindas do câncer e tratamento oncológico, o qual modificou o perfil do estado nutricional medido pelo IMC nesta população de pacientes. Assim, antes da doença e tratamento, a prevalência de pacientes na condição de eutróficos era de 35\%, e na condição de sobrepeso/obesidade era de 65\%. Não havia, por esse critério, pacientes com quadro de desnutrição. Atualmente, mesmo após concluído o tratamento oncológico, observa-se que 10\% dos pacientes não recuperaram seu estado nutricional inicial, estando em desnutrição 45\% dos que foram classificados como eutróficos após tratamento e 35\% com sobrepeso. A mudança do perfil nutricional desta população pode estar relacionada à aplicação concomitante de várias modalidades no tratamento oncológico e doença avançada. ${ }^{29-32}$

De fato, observa-se nesta população a aplicação de várias modalidades terapêuticas de tratamento, como radioterapia (aplicada em 100\% dos pacientes), quimioterapia (55\%) e cirurgia (60\%), além de elevada prevalência de doença oncológica avançada, com 75\% dos pacientes apresentando doença classificada nos estadiamentos graus III e IV. Estes achados são semelhantes a outros trabalhos que descrevem a diminuição de até 4\% no IMC, além da diminuição de massa muscular e massa gorda em pacientes politratados que concluíram o tratamento oncológico no mínimo há seis meses. Isso demonstra a magnitude dos efeitos do tratamento oncológico no estado nutricional desses indivíduos e a extensão do tratamento oncológico. ${ }^{30,33-35}$

Nesta população, não foram verificados pacientes com baixo IMC antes do tratamento oncológico. Estudos demonstram que um baixo IMC antes do tratamento oncológico está implicado, ainda, com menor taxa de sobrevida livre de doença. ${ }^{29,33-34}$

A ferramenta ASG-PPP apresenta adequada sensibilidade e especificidade para diagnosticar o estado nutricional de pacientes oncológicos, estando validada para essa população. ${ }^{36,37}$ Neste ensaio, 80\% dos pacientes, mesmo após concluído tratamento, apresentavam risco de desnutrição ou desnutrição moderado. Esta prevalência é semelhante à de outros estudos que observaram que, após a quimiorradioterapia, a desnutrição pode aumentar em até 85\%.33,38 
Estudo multicêntrico com pacientes internados portadores de câncer em cabeça e pescoço realizado no Brasil identificou por intermédio da ASG-PPP que há um risco de inadequação do estado nutricional de $(O R 3,7)$ para essa população. Neste estudo, verificou-se também que o sintoma único de disfagia foi fator independente para má condição nutricional e também de sobrevida (OR 2,75), demonstrando a necessidade de controle desse sintoma pelas equipes multidisciplinares. ${ }^{39}$ De modo semelhante, no presente estudo, sintomas de xerostomia, disfagia e disgeusia foram os mais prevalentes.

Em relação aos escores de adesão nutricional às recomendaç̃̃es WCRF / IARC, observou-se baixa adesão dos

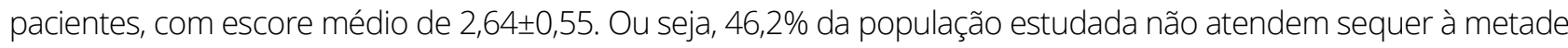
das recomendações WCRF / IARC para alimentação e peso corporal saudável. Somente a diretriz de redução do consumo de álcool foi totalmente atendida por essa população.

A adesão às recomendações de alimentação, nutrição, atividade física e peso saudável pode prevenir um entre cada quatro novos casos de câncer. 10,40 Estudo semelhante, avaliando o grau de adesão às recomendaç̃̃es WCRF /

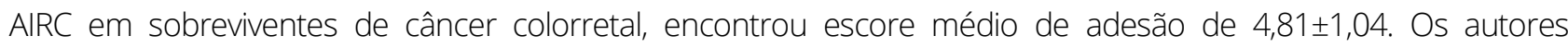
observaram que escores mais altos de adesão às recomendações WCRF / AICR foram associados a melhor estado de saúde global, função física, função cognitiva, função social e menor fadiga, além de menor recorrência de tumores. $^{41}$

Em estudo longitudinal realizado com pacientes chineses com câncer de mama, os autores verificaram maior adesão às recomendações WCRF / AICR após o diagnóstico de câncer, aumentando de 3,2 21,1 (antes do tratamento)

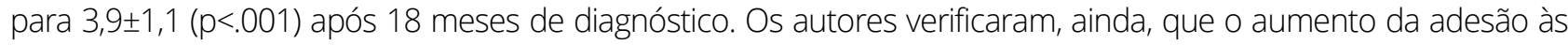
recomendações WCRF / AICR após o diagnóstico de câncer estava relacionado ao melhor escore para a qualidade de vida. 42,43

Até o presente momento, este é o primeiro estudo relativo à adesão às recomendaç̃̃es WCRF / IARC para SCCO, que avaliam também o estado nutricional e a sintomatologia apresentados por essa população. Por meio deste ensaio, pode-se verificar as limitações em termos de atendimento às recomendações de alimentação saudável que sofrem SCCO. O consumo prevalente de carne processada e bebidas açucaradas, e o baixo consumo de vegetais em geral estão relacionados aos casos de sobrepeso/obesidade. Trata-se de fator de risco para um segundo tumor, já que provoca um processo inflamatório crônico e altera o metabolismo de hormônios, provocando danos celulares e favorecendo o surgimento da doença. Uma alimentação saudável combinada com atividade física regular ajuda a controlar o peso corporal. ${ }^{41}$ Uma alimentação rica em frutas, verduras e legumes é fator de proteção para CCO. ${ }^{44}$ Na população estudada, somente três pacientes estavam em atendimento nutricional (dados não apresentados).

Apesar de a atividade física não ter sido mensurada neste trabalho, chama a atenção um dado verificado pela ASG-PPP, que demonstra que 75\% dos pacientes deste estudo não se sentem bem para realizar suas atividades habituais, ficando mais da metade do dia deitados ou sentados. Essa indisposição para atividades em geral pode contribuir para o sedentarismo, aumentando o fator de risco para um segundo tumor. ${ }^{42}$

Este estudo, até o presente momento, é o primeiro no Brasil que avalia o grau de atendimento às recomendações WCRF / AICR de alimentação e peso corporal através de um escore numérico em pacientes sobreviventes de câncer de cavidade oral. Identificamos, entretanto, limitações que estão relacionadas ao tipo de estudo transversal, potencial viés na seleção amostral, uma vez que esses pacientes eram derivados de outro estudo com amostra selecionada por conveniência. Observa-se heterogeneidade dos tipos de câncer de cavidade oral e número amostral pequeno. Essas limitações não permitem estabelecer associações entre todas as variáveis relacionadas com a baixa adesão às recomendações; assim, seria necessário realizar estudos prospectivos de longo prazo para verificar tais associações. 


\section{CONCLUSÃO}

Por meio deste estudo, verificou-se que grande parte dos pacientes desta amostra de SCCO apresentam risco de desnutrição ou estão com desnutrição moderada. Foi observado alto percentual de sintomatologias que impactaram na ingestão alimentar e na adesão às recomendações WCRF / AICR. O perfil alimentar foi marcado por elevado consumo de carnes processadas, bebidas açucaradas e baixo consumo de alimentos de proteção para cCO.

A partir desses dados, sugere-se o estabelecimento de novos protocolos de pesquisa, a fim de correlacionar os fatores intervenientes na baixa adesão às recomendações WCRF / AICR, guiando assim formulações de novos protocolos clínicos que garantam a ingestão alimentar adequada para a prevenção de novos tumores.

\section{REFERÊNCIAS}

1. AICR, WCRF. Survivors of breast and other cancers 2018. [Acesso em 13 fev 2019]. Disponível em: https://www.wcrf.org/sites/default/files/Cancer-Survivors.pdf

2. Jemal LT, Soerjomataram FBI, Adams CA. The Cancer Atlas 4, Third Edition. [Acesso em 20 mar 2020]. Disponível em: https://www.cancer.org DOI: 10.3322/caac.21590

3. Ministério da Saúde, Instituto Nacional Câncer. Estimativa 2020 Incidência de câncer no Brasil. Instituto Nacional de Câncer José Alencar Gomes da Silva; 2019.130 p

4. Hutcheson KA, Eisbruch A, Beitler JJ, Langendijk JA, Lee AWM, Cory J, et al. Treatment of late sequelae after radiotherapy for head and neck cancer. Cancer Treat Rev. 2017; 59:79-92 doi: 10.1016/j.ctrv.2017.07.003.

5. Freitas D.A, Caballero AD, Pereira MM, Oliveira SKM, Silva GPE, Hernández CIV. Sequelas bucais da radioterapia de cabeça e pescoço. Rev CEFAC. 2011; 13(6):1103-1108; https://doi.org/10.21270/archi.v8i12.4800

6. Nora T, Chan D, Lau R, Continuous update of the WCRF-AICR report on diet and cancer. 2007;1-50.

7. Er V, Lane JA, Martin RM, Emmett P, Gilbert R, Avery KN, Walsh E, Donovan JL, Neal DE, Hamdy et at, Adherence to dietary and lifestyle recommendations and prostate cancer risk in the prostate testing for cancer and treatment (ProtecT) trial. Cancer Epidemiology Biomarkers and Prevention. 2014; 23(10)-doi: 10.1158/1055-9965.EPI-14-0322.

8. Jankovic N, Geelen A, Winkels RM, Mwungura B, Fedirko V, Jenab M, Iner AK, Brenner H, Ordóñez-Mena et a; Adherence to the WCRF/AICR dietary recommendations for cancer prevention and risk of cancer in elderly, the chances consortium. Eur J Epidemiol. 2015; 26 (1): 136-144 doi: 10.1158/1055-9965

9. Romaguera D, Vergnaud AC, Peeters PH, Van Gils CH, Chan DSM, Ferrari P, et al. Is concordance with World Cancer Research Fund/American Institute for Cancer Research guidelines for cancer prevention related to subsequent risk of cancer? Results from the EPIC study. Am J Clin Nutr. 2012; 96(1):150-6 doi: 10.3945/ajcn.111.0316743.

10. Harris HR, Bergkvist L, Wolk A. Adherence to the World Cancer Research Fund/American Institute for Cancer Research recommendations and breast cancer risk. Int J Cancer. 2016; 138(11):2657-64. doi: 10.1002/ijc.30015

11. Chao-Seijo B, Arza-Ovejas V, Lertxundi-Alegria I, Aguirre C, Bujanda L, Fernandez F P, Portillo I, et al. Adherence to guidelines for colorectal cancer prevention and its relationship to this cancer in the Basque country: A case-control study. Annals of Oncology 2016; y 27 (Supplement 6): vi149-vi206-doi.org/10.1093/annonc/mdw370.59

12. Hofto S, Abbott J, Jackson JE, Isenring E. Investigating adherence to Australian nutritional care guidelines in patients with head and neck cancer. Cancers Head Neck. 2018; Dec 3(1). doi: 10.1186/s41199-018-0033-9.

13. Crowder SL, Douglas KG, Yanina PM, Sarma KP, Arthur AE. Nutrition impact symptoms and associated outcomes in post-chemoradiotherapy head and neck cancer survivors: a systematic review. Journal of Cancer Survivorship. 2019; 18, 57.doi.org/10.1186/s12937-019-0479-6

14. Oliver JR, Wu SP, Chang CM, Roden DF, Wang B, Hu KS, et al. Survival of oral tongue squamous cell carcinoma in young adults. Head Neck. 2019; 41(9):2960-8. doi: 10.1002/hed.25772. 
15. Rocha BQC, Eneas L, Oliveira RG, Junqueira RB, Verner FS. Características epidemiológicas de pacientes portadores de neoplasias de cabeça e pescoço submetidos à radioterapia em Juiz de Fora - MG. HU Rev. 2017; $43: 2644$. doi.org/10.34019/1982-8047

16. Borges LR, Gonzalez MC, Paiva SI, Silveira DH, Assunção MCF. Can nutritional status influence the quality of life of cancer patients? Rev Nutr. 2010; 23:5; https://doi.org/10.1590/S1415-52732010000500005

17. Ottery FD. Definition of standardized nutritional assessment and interventional pathways in oncology. Nutrition [Internet]. 1996 Jan 1; 12(1):S15-9. doi: 10.1016/0899-9007(96)90011-8

18. Gonzalez MC, Borges LR, Silveira DH, Assunção MCF, Orlandi SP. Validação da versão em português da avaliação subjetiva global produzida pelo paciente Validation of a Portuguese version of patient-generated subjective global assessment. Rev Bras Nutr Clin. 2010; 25(2):102-8.

19. World Health Organization: WHO Physical status: the use and interpretation of anthropometry. Report of a WHO Expert Committee. World Health Organ Tech Rep Ser.; 1995. [Acesso em 13 mai 2020] Disponível em https://apps.who.int/iris/bitstream/handle/10665/37003/WHO_TRS_854.pdf;jsessionid=86A71E6479E45480A686A B9F43454B2A?sequence $=1$

20. Crispim SP, Fisberg RM, Almeida CC, Bettega N, Geneviève KV, Pereira RA, Marchiori DML, Santos N A, Steluti J, Slimani N. Manual fotográfico de quantificação alimentar. Curitiba: Universidade Federal do Paraná; 2017.147 p.: il.: colorido 2017. [Acesso em 05 fev 2019]. Disponível em: http://www.ucv.edu.br/biblioteca/livro-virtual/manual-fotograficoquantificacao-alimentar.pdf https://doi.org/10.1590/1980-549720200013

21. Ministério da Saúde. Consenso Nacional de Nutrição Oncológica. Instituto Nacional de Câncer José Alencar Gomes da Silva (INCA). [Acesso em 13 maio 2020]. Disponível em: https: www.inca.gov.br

22. Shams-White MM, Brockton NT, Mitrou P, Romaguera D, Brown S, Bender A, et al. Operationalizing the 2018 World Cancer Research Fund / American Institute for Cancer Research (WCRF / AICR) Cancer Prevention Recommendations: A standardized scoring system. Nutrients.; 2019 Jul 1; 11(07) doi: 10.3390/nu11071572.

23. Freitas RM, Rodrigues MX, Júnior A F M, Oliveira GAL. Fatores de risco e principais alterações citopatológicas do câncer bucal uma revisão de literatura. Rev Bras Anal Clin [Internet]. 2016: 48(01):13-8.

24. Oliveira FP, Santos A, Viana MS, Alves JL, Pinho NB, Reis PF. Perfil Nutricional de Pacientes com Câncer de Cavidade Oral em Pré-Tratamento Antineoplásico. Rev Bras Cancerol. 201: 61(3):253-9. doi: https://doi.org/10.32635/21769745.RBC.2015v61n3.255

25. Gorenc M, Kozjek NR; Malnutrition and cachexia in patients with head and neck cancer treated with (chemo) radiotherapy. 2015; 0:249-58 doi: 10.1016/j.rpor.2015.03.001.

26. Tseng KS, Lin C, Lin YS, Weng SF. Risk of head and neck cancer in patients with diabetes mellitus: A retrospective cohort study in Taiwan. JAMA Otolaryngol - Head Neck Surg. 2014: 140(8):746-53 doi:10.1001/jamaoto.2014.1258.

27. Wang X, Wang H, Zhang T, Cai L, Dai E, He J. Diabetes and its potential impact on head and neck oncogenesis. J Cancer. 2020; 11(3):583-91 doi:10.7150/jca.35607.

28. Body Q. Characterization M, Head A, Using F, Tomography C, Presland RB, et al. A retrospective study of 606 cases of nasopharyngeal carcinoma with or without oropharyngeal candidiasis during radiotherapy. PLoS One [Internet]. 2017; 10(2):1-10 doi.org/10.1038/bjc.2017.138

29. Li Z, Zou L, Liu T, Yang A. Prognostic value of body mass index before treatment for laryngeal squamous cell carcinoma. 2015:394-400 doi: 10.7497/:

30. Gellrich N, Handschel J, Holtmann H, Krüskemper G. Oral Cancer Malnutrition Impacts Weight and Quality of Life. 201:2145-60. doi: 10.3390/nu7042145

31. Jung AR, Roh JL, Kim JS, Kim SB, Choi SH, Nam SY, et al. Prognostic value of body composition on recurrence and survival of advanced-stage head and neck cancer. Eur J Cancer. 2019: Jul 1; 116:98-106. doi: 10.1016/j.ejca.2019.05.006.

32. Ottosson S, Söderström K, Kjellén E, Nilsson P, Zackrisson B, Laurell G. Weight and body mass index in relation to irradiated volume and to overall survival in patients with oropharyngeal cancer: A retrospective cohort study. 2014:18. doi: 10.1186/1748-717X-9-160 
33. Orell $H$, Schwab U, Saarilahti K, Österlund P, Ravasco P, Mäkitie A. Nutritional counseling for head and neck cancer patients undergoing (chemo) radiotherapy: a prospective randomized trial. Front Nutr. 2019; Mar 18;6. doi: 10.3389/fnut.2019.00022.

34. Chamchol S, Fuller CD, Mohamed ASR, Grossberg A, Messer JA, Heukelom J, et al. Quantitative body mass characterization before and after head and neck cancer radiotherapy: A challenge of height-weight formulae using computed tomography measurement. Oral Oncol. 2016: Oct 1; 61:62-9 doi: 10.1016/j.oraloncology.2016.08.012.

35. Pedroso P, Medeiras F, Estevam Martinez C, Silva Cardoso S. Estado Nutricional e Ingestão Alimentar de Pacientes com Câncer de cabeça e pescoço submetidos a tratamento oncológico Arq. Ciênc. Saúde. 2016; out-dez (23). Disponível em: https://doi.org/10.17696/2318-3691.23.4.2016.480

36. Campos MB, Peixoto ARB, Alves MM, Carvalho APPF, Carvalho NDM. Avaliação Nutricional De Pacientes OncoHematológicos em Quimioterapia Suplementados Com Glutamina. Rev Bras Ciências da Saúde. 2016; 20(4):319326.

37. Medeiras FPP, Martinez CE, Cardoso SDS. Estado nutricional e ingestão alimentar de pacientes com câncer de cabeça e pescoço submetidos a tratamento oncológico. Arq Ciências da Saúde. 2016; dez [S.I.] 23(4):43-47. doi: https://doi.org/10.17696/2318-3691.23.4.2016.480

38. Pinho NB, Martucci RB, Rodrigues VD, D'Almeida CA, Thuler LCS, Saunders, C, et al. Malnutrition associated with nutrition impact symptoms and localization of the disease: Results of a multicentric research on oncological nutrition. Clin Nutr. 2019; Jun 1 38(3):1274-9. doi: 10.1016/j.clnu.2018.05.010

39. Skolarus TA, Zhang $Y$, Hollenbeck BK. Understanding fragmentation of prostate cancer survivorship care: Implications for cost and quality. Cancer. 2012; 118(11):2837-45. doi: 10.1002/cncr.26601.

40. Van Veen MR, Mols F, Bours MJL, Weijenberg MP, Kampman E, Beijer S. Adherence to the World Cancer Research Fund / American Institute for Cancer Research recommendations for cancer prevention is associated with better health-related quality of life among long-term colorectal cancer survivors: results of the PROFILES registry. Support Care Cancer. 2019 Dec 1; 27(12):4565-74. doi: 10.1007/s00520-019-04735-y.

41. Romaguera D, Lavedan EG, Molinuevo A, Batlle J, Mendez AN, Moreno V, Vidal C, Castelló A, Pérez-Gómez B, et al. Adherence to nutrition-based cancer prevention guidelines and cancer risk in the MCC-Spain case-control study. Ann Nutr Metab. 2015; 26: 277-86. doi:10.1002/ijc.30722

42. Fanidi A, Ferrari P, Biessy C, Ortega C, Angeles-Llerenas A, Torres-Mejia G, et al. Adherence to the World Cancer Research Fund / American Institute for Cancer Research cancer prevention recommendations and breast cancer risk in the Cancer de Mama (CAMA) study. Public Health Nutr. 2015; 18 (18):3337-3348. doi: $10.1017 /$ S1368980015000634

43. Lang S, Schimansky S, Beynon R, Penfold C, Davies A, Waylen A, et al. Dietary behaviors and survival in people with head and neck cancer: Results from Head and Neck 5000. Head Neck. 2019 Jul 1;41(7):2074-84. doi: 10.1002/hed.25660.

44. Cohen EEW, LaMonte SJ, Erb NL, Beckman KL, Sadeghi N, Hutcheson KA, et al. American Cancer Society Head and Neck Cancer Survivorship Care Guideline. CA Cancer J Clin. 2016; 66(3):203-39. doi: 10.3322/caac.21343

\section{Colaboradoras}

Bezerra GB contribuiu com a coleta dos dados, analise, concepção dos dados, interpretação dos dados revisão e aprovação da versão final. Rodrigues CF contribuiu para a concepção e desenho do estudo, interpretação dos dados, revisão e aprovação da versão final. Martucci RB contribuiu com analise e interpretação dos dados, revisão e aprovação da versão final.

Conflito de Interesses: As autoras declaram não haver conflito de interesses.

Recebido: 05 de maio de 2020

Aprovado: 15 de setembro de 2020 